\title{
QUALIDADE DE MANGAS CV. PALMER APÓS ARMAZENAMENTO SOB BAIXAS TEMPERATURAS ${ }^{1}$
}

\author{
ANA CAROLINA ALMEIDA MIGUEL 2 , JOSÉ FERNANDO DURIGAN ${ }^{3}$, \\ JOSÉ CARLOS BARBOSA ${ }^{4}$, CRISTIANE MARIA ASCARI MORGADO
}

RESUMO - Este trabalho objetivou avaliar a qualidade de mangas 'Palmer' previamente armazenadas em baixas temperaturas, após sua transferência para a condição de ambiente. Frutos colhidos no estádio de maturação fisiológica foram cuidadosamente transportados, selecionados, padronizados quanto à coloração, tamanho e ausência de injúrias, e tratados com fungicida, antes de serem armazenados a $2^{\circ} \mathrm{C}(75,7 \% \mathrm{UR})$, $5^{\circ} \mathrm{C}(73,8 \%$ UR $)$ e $12^{\circ} \mathrm{C}(82 \%$ UR $)$, por $7 ; 14$ e 21 dias. Ao final de cada período, os frutos foram transferidos para temperatura ambiente $\left(22,9^{\circ} \mathrm{C} ; 62,3 \% \mathrm{UR}\right)$, onde foram mantidos por $1 ; 3 ; 5$ e 7 dias, simulando o período de comercialização, e avaliados quanto à ocorrência de injúrias e podridões, coloração da casca e polpa, firmeza da polpa, teores de sólidos solúveis, acidez titulável e de ácido ascórbico, além da atividade das enzimas peroxidase, polifenoloxidase e fenilalanina amônia-liase. Os resultados indicaram que as mangas 'Palmer' podem ser conservadas a $12^{\circ} \mathrm{C}$ por 21 dias, sem prejuízos ao amadurecimento, porém com limitações devido à ocorrência de podridões. $\mathrm{O}$ armazenamento a $2^{\circ} \mathrm{C}$ e a $5^{\circ} \mathrm{C}$ foi limitado pela ocorrência de injúrias na casca, porém na temperatura de $2^{\circ} \mathrm{C}$ estes sintomas foram mais severos e comprometeram o desenvolvimento da coloração característica da casca. Entretanto, o amadurecimento da polpa destes frutos não foi prejudicado, mas este processo ocorreu com menor intensidade que nas mangas mantidas a $12^{\circ} \mathrm{C}$.

Termos para indexação: Mangifera indica, injúria pelo frio, conservação, enzimas, podridão.

\section{QUALITY OF MANGOES CV. PALMER AFTER THEIR STORAGE UNDER LOW TEMPERATURES}

\begin{abstract}
This study aimed to evaluate the quality of 'Palmer' mangoes previously stored at low temperatures, after their transference to the environmental condition. Fruits harvested at physiological maturity were carefully transported to the Laboratory where they were selected, standardized as the color, size and absence of injuries and treated with fungicide before they were stored at $2^{\circ} \mathrm{C}(75.7 \% \mathrm{RH}), 5^{\circ} \mathrm{C}(73.8 \% \mathrm{RH}) \mathrm{e}$ $12^{\circ} \mathrm{C}(82 \% \mathrm{RH})$ for 7,14 and 21 days. At the end of each period, the fruits were transferred to environmental condition $\left(22.9^{\circ} \mathrm{C} ; 62.3 \% \mathrm{RH}\right)$, where they were kept for $1,3,5$ and 7 days, simulating the trading period, and evaluated for the occurrence of injuries and rottenness; peel and pulp color; firmness; contents of soluble solids, titratable acidity and ascorbic acid, as well as, the activities of the enzymes peroxidase, polyphenoloxidase and phenylalanine ammonia-lyase. The results indicated that 'Palmer' mangoes can be stored at $12^{\circ} \mathrm{C}$ for 21 days without damage to ripening, but with limitations due to the occurrence of decay. The storage at $2^{\circ} \mathrm{C}$ and $5^{\circ} \mathrm{C}$ was limited by the occurrence of injuries in the peel, but at the temperature of $2^{\circ} \mathrm{C}$ these symptoms were more severe and compromised the development of the characteristic color of the peel. However, the ripening of the pulp was not harmed, but this process occurred with less intensity than in mangoes maintained at $12^{\circ} \mathrm{C}$.
\end{abstract}

Index terms: Mangifera indica, chilling injury, conservation, enzymes, decay.

\footnotetext{
1(Trabalho 155-12). Recebido em: 16-05-2012. Aceito para publicação em: 07-06-2013.

${ }^{2}$ Eng $^{\mathrm{a}} \mathrm{Agr}^{\mathrm{a}}$, Doutoranda em Agronomia, Depto. Tecnologia, Via de Acesso Prof. Paulo Donato Castellane, s/n 14884-900, JaboticabalSP. E-mail: anaamiguel@yahoo.com.br

${ }^{3}$ Prof $^{0}$ do Departamento de Tecnologia da FCAV/UNESP, Jaboticabal. E-mail: jfduri@fcav.unesp.br

${ }^{4}$ Prof $^{\mathrm{o}}$ do Departamento de Ciências Exatas da FCAV/UNESP, Jaboticabal. E-mail: jcbarbosa@fcav.unesp.br

${ }^{5}$ Doutoranda em Agronomia da FCAV/UNESP; Jaboticabal-SP. E-mail: cristianemorgado4@yahoo.com.br
} 


\section{INTRODUÇÃO}

A manga (Mangifera indica L.) é um fruto tipicamente tropical, com sabor e aroma atrativos, que conquistou a preferência de consumidores, sendo considerado de relevante expressividade no agronegócio internacional. O Brasil ocupa a terceira colocação no ranking dos exportadores de mangas in natura, e, ao mesmo tempo, esta fruta é a terceira mais exportada pelo País (PINTO, et al., 2011) demonstrando sua importância e seu grande potencial para exportação (MAPA, 2011).

Dentre as variedades exploradas, destacam-se a 'Tommy Atkins', a 'Palmer', a 'Kent' e a 'Keitt' (MENTEN, 2010). Todavia, a 'Palmer' tem crescido em importância, por ser uma variedade tardia, bem aceita no mercado interno, que apresenta boa capacidade de conservação e boas perspectivas para exportação (TEIXEIRA et al., 2011).

$\mathrm{O}$ armazenamento refrigerado é uma ferramenta importante para o prolongamento da vida útil de frutos. Possibilita a comercialização em locais distantes da produção, usando-se meios de transporte mais demorados, cujo frete tem custo, geralmente, menor, melhorando a competitividade no mercado internacional (LIMA et al., 2006). Todavia, em frutos de origem tropical, a refrigeração pode levar ao aparecimento posterior de distúrbios fisiológicos que afetam negativamente sua qualidade, assim que eles são removidos desta condição.

Em mangas, a sensibilidade ao frio tem limitado a extensão de seu período de armazenamento, comercialização e transporte (KADER; ARPAIA, 2002). Temperaturas inferiores a $7-13^{\circ} \mathrm{C}$, dependendo do estádio de maturação e da cultivar, resultam na formação de pontuações necróticas e de regiões deprimidas e descoloridas em sua epiderme. Além disso, outros sintomas como enrijecimento da polpa, ausência de aroma característico e amadurecimento anormal também são evidenciados (NAIR; SINGH, 2009). Nunes et al. (2007) indicaram que $12^{\circ} \mathrm{C}$ é a temperatura recomendada para o armazenamento de mangas 'Palmer' colhidas no estádio “de vez" ou com mais de $50 \%$ da casca com cor vermelha ou amarela, sem risco da ocorrência de danos pelo frio.

A breve exposição de um tecido à condição que provoca injúria pelo frio pode não ocasionar o desenvolvimento dos sintomas, enquanto ele é mantido sob refrigeração, os quais aparecerão após sua remoção desta condição e exposição a temperaturas mais elevadas (CHITARRA; CHITARRA, 2005). De maneira geral, quanto mais baixa a temperatura de armazenamento, menor o tempo de exposição necessário para desencadear o desenvolvimento destes sintomas (CANTWELL; KASMIRE, 2002).

Este trabalho objetivou avaliar a qualidade de mangas 'Palmer' após seu armazenamento refrigerado.

\section{MATERIAL E MÉTODOS}

Mangas 'Palmer' colhidas no estádio de maturação fisiológica ou entre os graus 2 e 3 (ALVES et al., 2002), em pomar comercial localizado no município de Monte Alto-SP, foram transportadas cuidadosamente, por $20 \mathrm{~km}$, até o Laboratório de Tecnologia dos Produtos Agrícolas da FCAV/UNESP. $\mathrm{Na}$ recepção, seus pedúnculos foram padronizados em 10-20 mm, e elas foram lavadas com detergente neutro, enxaguadas em água corrente e novamente selecionadas, eliminando-se as desuniformes. Em seguida, estes frutos foram tratados por imersão em fungicida Magnate $500 \mathrm{EC}^{\circledR}$ (Imazalil a 50\%) a $200 \mathrm{~mL} \cdot 100 \mathrm{~L}^{-1}$, a $10^{\circ} \mathrm{C}$ por 2 minutos (SANTOS et al., 2010), e armazenados a $2 \pm 0,4^{\circ} \mathrm{C}(75,7 \pm 15,6 \%$ UR), $5 \pm 1^{\circ} \mathrm{C}(73,8 \pm 12,8 \%$ UR $)$ e $12 \pm 0,9^{\circ} \mathrm{C}(82 \pm 9,7 \%$ UR) por 7; 14 e 21 dias. Ao final de cada período, foram transferidos para condição ambiente $\left(22,9^{\circ} \mathrm{C}\right.$; $62,3 \%$ UR), na qual foram mantidos por $1 ; 3 ; 5 \mathrm{e}$ 7 dias, simulando o período de comercialização. Utilizou-se delineamento inteiramente casualizado, contendo 36 tratamentos (3 períodos de armazenamento refrigerado $\times 3$ temperaturas de refrigeração x 4 períodos de armazenamento à temperatura ambiente), com três repetições constituídas de dois frutos cada.

Os frutos foram avaliados quanto à ocorrência de injúrias pelo frio ou chilling, através de observação visual e atribuição de notas, baseando-se no proposto por Whangchai et al. (2000): $8=$ severo $(>55 \%$ da superfície dos frutos contendo depressões e escaldadura); $7=$ forte ( $45-55 \%$ da superfície injuriada); $6=$ moderado/forte ( $35-45 \%$ da superfície injuriada); $5=$ moderado $(25-35 \%$ da superfície injuriada); $4=$ suave $(15-25 \%$ da superfície injuriada); $3=$ brando (5-15\% da superfície injuriada); $2=$ leve $(2-5 \%$ da superfície danificada); $1=$ sem sintomas visíveis de injúria. Considerou-se a nota 5 como o valor-limite para comercialização.

A incidência de podridões foi avaliada visualmente, contando-se o número de mangas com presença de podridões, com diâmetro superior a 0,5 $\mathrm{cm}$, sendo os resultados expressos em porcentagem.

A coloração da casca e da polpa foi determinada utilizando-se colorímetro Minolta CR 400, pela média das medições, em dois pontos opostos na região equatorial do fruto, com os resultados expressos em luminosidade $\left(L^{*}\right)$, ângulo hue $\left({ }^{\circ} \mathrm{Hue}\right)$ e cromati- 
cidade (Croma). A firmeza da polpa foi determinada utilizando-se de penetrômetro, aplicado na mesma região em que a variável coloração foi mensurada, sendo os resultados expressos em Newton (N).

$\mathrm{Na}$ polpa, foram determinados os teores de sólidos solúveis (SS) e acidez titulável (AT), de acordo com a AOAC (2005), além dos teores de ácido ascórbico (STROHECKER; HENNING, 1967). Também se quantificou a atividade das enzimas polifenoloxidase (PPO) e peroxidase (POD), utilizando-se do método indicado por Teixeira et al. (2007) para a PPO, e o indicado por Lima et al. (1999), para a POD. A atividade da fenilanalina amônia-liase (PAL) foi determinada usando-se método adaptado de Cahill e McComb (1992).

Os resultados foram analisados em esquema fatorial $3 \times 3 \times 4$, em que o primeiro fator correspondeu às temperaturas de armazenamento $\left(2^{\circ} \mathrm{C}, 5^{\circ} \mathrm{C}\right.$ e $12^{\circ} \mathrm{C}$ ); o segundo, ao tempo de armazenamento refrigerado ( $7 ; 14$ e 21 dias), e o terceiro, ao período de armazenamento sob condição de ambiente $(1 ; 3 ; 5$ e 7 dias). Os dados foram submetidos à análise de variância pelo teste $\mathrm{F}$, comparação das médias pelo teste de Tukey, ao nível de $1 \%$ e de $5 \%$ de probabilidade, e regressão polinomial para os fatores quantitativos.

\section{RESULTADOS E DISCUSSÃO}

Observou-se que, após a transferência para temperatura ambiente, os frutos mantidos a $2^{\circ} \mathrm{C}$ ou $5^{\circ} \mathrm{C}$, por 7 dias, já estavam inaptos à comercialização (Tabela 1), dada a ocorrência de injúria por frio, de forte (nota 7) a moderado/forte (nota 6). As mangas armazenadas a $2^{\circ} \mathrm{C}$ ou $5^{\circ} \mathrm{C}$ apresentavam, após 14 dias, danos severos (nota 8). Efeito semelhante foi observado por Nunes et al. (2007), que descreveram agravamento dos sintomas, em mangas desta cultivar, em dois dias após sua transferência para a condição de ambiente.

Verificou-se que quanto maior a temperatura de armazenamento e mais extenso o período de refrigeração e ao ambiente, maior foi a incidência de podridões (Tabela 2), o que limitou a conservação dos frutos mantidos a $12^{\circ} \mathrm{C}$, ainda durante o armazenamento refrigerado (Figura 1A). Em mangas da mesma cultivar, mantidas a $12^{\circ} \mathrm{C}(65 \%$ UR) por 21 dias, Santos (2008) não detectou esta incidência de sintomas, o que foi atribuído à menor contaminação inicial.

A luminosidade da casca (L_c) foi significativamente afetada pela temperatura do armazenamento refrigerado e pelo tempo sob refrigeração (Tabela 2). Os frutos previamente mantidos sob condição de injúria pelo frio $\left(2^{\circ} \mathrm{C}\right.$ e $\left.5^{\circ} \mathrm{C}\right)$ exibiram casca mais escurecida que os conservados a $12^{\circ} \mathrm{C}$, o que pode ser atribuído à ocorrência dos sintomas de danos por chilling, que se caracterizam pela formação de manchas escuras na casca (CHITARRA; CHITARRA, 2005). Analisando o efeito da interação entre as temperaturas de refrigeração e os períodos de armazenamento refrigerado, constatou-se que somente os frutos mantidos a $12^{\circ} \mathrm{C}$ apresentaram aumento significativo nos valores de luminosidade, o que reforça a ausência de visualização de danos para esta temperatura.

O ângulo Hue da casca (Hue_c) foi influenciado pela temperatura e pelo tempo sob refrigeração e caracterizou- se por aumento nos valores com o período de conservação, indicando que a casca se tornou vermelho-amarelada, com intensidades diferentes (Tabela 2). Este efeito, que também foi observado por Nunes et al. (2007) em mangas 'Tommy Atkins' e 'Palmer', tem sido atribuído ao amadurecimento, que ocorre mais rapidamente quando elas são armazenadas em temperaturas acima de $5^{\circ} \mathrm{C}$.

A cromaticidade da casca (Croma c) foi significativamente influenciada pela temperatura, pelo tempo de refrigeração e pelo período de conservação ao ambiente (Tabela 2). Os frutos previamente armazenados a $12^{\circ} \mathrm{C}$ apresentaram aumento expressivo na síntese de pigmentos ao longo dos 21 dias de armazenamento refrigerado, cuja tendência continuou após sua transferência à temperatura ambiente, e é característica do amadurecimento (Figuras $1 \mathrm{~B} ; 1 \mathrm{C})$. Os conservados a $2^{\circ} \mathrm{C}$ tiveram a síntese de pigmentos da casca significativamente reduzida durante o armazenamento refrigerado, e não tiveram esta atividade retomada quando levados ao ambiente. Durante o armazenamento a $5^{\circ} \mathrm{C}$, esta síntese não se alterou significativamente, porém foi retomada após os frutos serem levados ao ambiente, mas com menor intensidade que a $12^{\circ} \mathrm{C}$.

A análise conjunta das variáveis que compõem a coloração da casca indicou que as mangas armazenadas a $12^{\circ} \mathrm{C}$ se caracterizaram pela cor vermelho-brilhante, a qual foi diferente da observada nas mantidas a $2^{\circ} \mathrm{C}$ (esverdeada) e $5^{\circ} \mathrm{C}$ (vermelho-amarelada escurecido). A coloração observada também indica a perda de qualidade, devido à injúria pelo frio (CHITARRA; CHITARRA, 2005; MIGUEL et al., 2011).

Na polpa, a luminosidade ( $\left.L \_p\right)$ só foi significativamente influenciada pela temperatura de armazenamento refrigerado e pelo tempo de conservação à temperatura ambiente (Tabela 2). Mangas armazenadas a $12^{\circ} \mathrm{C}$ caracterizaram-se pelos menores valores, indicando ocorrência de escurecimento, o qual se prolongou após sua transferência para 
temperatura ambiente, enquanto as mantidas a $2^{\circ} \mathrm{C}$ e a $5^{\circ} \mathrm{C}$ apresentaram polpa mais clara e só escureceram depois de levadas ao ambiente (Figura 1D), sugerindo que o armazenamento nestas temperaturas retardou a síntese de pigmentos.

O ângulo Hue da polpa (Hue_p) foi influenciado pela temperatura no período refrigerado e pelo período de conservação sob refrigeração e ao ambiente (Tabela 2). Durante o armazenamento refrigerado, a polpa dos frutos mantidos a $2^{\circ} \mathrm{C}$ e $5^{\circ} \mathrm{C}$ caracterizou-se pela coloração creme, enquanto nos armazenados a $12^{\circ} \mathrm{C}$ a diminuição nos valores indica que ela se tornou amarela (Tabela 3). Após a transferência dos frutos à condição ambiente, independentemente da temperatura e do período sob refrigeração, a coloração da polpa tornou-se amarelo-alaranjada.

A refrigeração a $2^{\circ} \mathrm{C}$ retardou a produção de pigmentos da polpa (Tabela 2 ), em relação a $5^{\circ} \mathrm{C}$ e $12^{\circ} \mathrm{C}$, indicado pela cromaticidade (Croma $p$ ). Influenciados pelo período em temperatura ambiente, estes frutos aumentaram esta síntese, independentemente da temperatura e do tempo de conservação inicial (Figura 1E).

Os resultados relativos à coloração da polpa indicam que ela se desenvolveu normalmente sob a condição de ambiente, porém em ritmo inicialmente mais lento a $2^{\circ} \mathrm{C}$, indicando que os sintomas de injúria pelo frio se restringem à casca (MORAIS; ASSIS, 2004).

A firmeza da polpa foi significativamente influenciada pela temperatura de armazenamento e pelo tempo sob refrigeração e ao ambiente (Tabela 2). Frutos mantidos a $12^{\circ} \mathrm{C}$ apresentaram polpa mais macia a partir do $7^{\circ}$ dia de refrigeração (Tabela 3), como consequência da maior atividade enzimática (CHITARRA; CHITARRA, 2005). Durante o período de armazenamento refrigerado, a resistência da polpa nas mangas armazenadas a $2^{\circ} \mathrm{C}$ e $5^{\circ} \mathrm{C}$ aumentou, porém reduziu-se depois de transferidas à condição de ambiente, mas com menor intensidade que as mantidas a $12^{\circ} \mathrm{C}$. Estes resultados indicam que o amolecimento da polpa não foi prejudicado pela temperatura de armazenamento, ou seja, o aumento na firmeza, sintoma indicativo de amadurecimento irregular, só ocorreu durante o período refrigerado, o que também foi relatado por Nunes et al. (2007), em mangas 'Palmer'.

Analisando-se o efeito da interação entre o tempo sob refrigeração e o período de armazenamento ao ambiente, sobre o amolecimento da polpa, observou-se que à medida que se prolongou o tempo de refrigeração, independentemente da temperatura, o amolecimento da polpa ocorreu de forma mais lenta após a transferência das mangas à temperatura ambiente (Tabela 3).

Os teores de sólidos solúveis (SS) foram influenciados pela temperatura do armazenamento refrigerado e pelo tempo de conservação ao ambiente (Tabela 4), com os frutos armazenados a $12^{\circ} \mathrm{C}$ apresentando teores significativamente maiores que os mantidos a $2^{\circ} \mathrm{C}$ e a $5^{\circ} \mathrm{C}$ no $14^{\circ}$ e $21^{\circ}$ dia (Tabela 5). Após a transferência dos mesmos ao ambiente, os teores de sólidos solúveis aumentaram, independentemente da condição de armazenamento prévio e da duração do período refrigerado, apesar de os frutos armazenados a $12^{\circ} \mathrm{C}$ terem apresentado os maiores valores nos três primeiros dias, para depois se assemelhar aos armazenados a $2^{\circ} \mathrm{C}$ e a $5^{\circ} \mathrm{C}$. O aumento nos teores de sólidos solúveis é um indicativo de amadurecimento da polpa (BERNARDES-SILVA et al., 2003) e reafirma a observação de que os danos pelo frio se limitam à casca (MIGUEL et al., 2011).

Os três fatores estudados influíram significativamente na acidez titulável, indicando que os frutos armazenados a $12^{\circ} \mathrm{C}$ se apresentavam menos ácidos (Tabela 4) e caracterizaram-se pela diminuição nos níveis de acidez após os 14 dias de armazenamento refrigerado (Figura $1 \mathrm{~F}$ ). Comportamento semelhante foi verificado nos mantidos a $2^{\circ} \mathrm{C}$ e a $5^{\circ} \mathrm{C}$, porém em menor intensidade, o que é concordante com o reportado por Vasquez-Salinas e Lakshminarayana (1985). Estes frutos, depois de transferidos para condição de ambiente, apresentaram redução significativa nos teores de acidez, independentemente da condição de armazenamento prévio (Tabela 4).

Os teores de ácido ascórbico (AA) reduziram-se significativamente durante o armazenamento ao ambiente (Tabela 4), independentemente da temperatura e da duração do período de refrigeração (Figura $1 \mathrm{G})$. Estes resultados indicam que a transferência para a temperatura ambiente $\left(22,3^{\circ} \mathrm{C}\right)$ acelerou o metabolismo e o consumo de ácidos orgânicos, dentre os quais o ascórbico, em reações oxidativas, que caracterizam o processo de amadurecimento (NEVES et al., 2008).

A atividade da peroxidase (POD) foi influenciada pela duração dos períodos sob refrigeração e condição de ambiente (Tabela 4) e caracterizou-se por aumento crescente na polpa dos frutos previamente armazenados a $2^{\circ} \mathrm{C}$ e a $12^{\circ} \mathrm{C}($ Figura $1 \mathrm{H}), \mathrm{o}$ que está relacionado com o processo de amadurecimento e deterioração da polpa, de que esta enzima participa, catalisando reações oxidativas (CORRÊA et al., 2007).

Houve efeito significativo de todos os fatores estudados na atividade de fenilalanina amônia-liase 
(Tabela 4), com maior atividade a $5^{\circ} \mathrm{C}$. Esta atividade aumentou até o $14^{\circ}$ dia nos frutos armazenados a $2^{\circ} \mathrm{C}$ e a $5^{\circ} \mathrm{C}$ e manteve-se nos conservados a $12^{\circ} \mathrm{C}$, para então reduzir-se no $21^{\circ}$ dia (Tabela 6). Após a transferência ao ambiente, ocorreu diminuição na atividade na polpa dos frutos previamente armazenados a $12^{\circ} \mathrm{C}$, enquanto nos oriundos do armazenamento sob temperaturas mais baixas, este comportamento foi detectado somente no $7^{\circ}$ dia. Em relação ao efeito da interação entre os tempos de refrigeração e o ambiente, observou-se que as mangas armazenadas por 14 dias foram as que se destacaram pela maior atividade enzimática, cuja tendência se prolongou até o $5^{\circ}$ dia. Além disso, verificou-se também redução nesta atividade na polpa dos frutos previamente mantidos por 14 e 21 dias sob refrigeração, seguido de 7 e 5 dias de conservação ao ambiente, respectivamente.

$A$ atividade da polifenoloxidase (PPO) também foi afetada pela temperatura e pelos tempos sob refrigeração e ao ambiente (Tabela 4 ). A atividade desta enzima foi mais ativa na polpa dos frutos mantidos a $2^{\circ} \mathrm{C}$ e $5^{\circ} \mathrm{C}$ e aumentou durante o período refrigerado (Tabela 6) e após sua transferência à condição de ambiente. Em relação ao efeito do tempo ao ambiente e sob refrigeração, observou-se que, quanto mais extenso o armazenamento, maior a atividade da PPO, indicando intensificação da ocorrência de reações oxidativas. Resultados semelhantes foram relatados por Vela et al. (2003) em mangas 'Manila' previamente armazenadas a $6^{\circ} \mathrm{C}$ e a $12^{\circ} \mathrm{C}$, o que foi atribuído ao processo de amadurecimento (WANG, 1982).

TABELA 1 - Injúria pelo frio* em mangas 'Palmer' armazenadas a $2^{\circ} \mathrm{C}(75,7 \%$ UR), 5ㄷ (73,8\% UR) e $12^{\circ} \mathrm{C}(82 \%$ UR), por até 28 dias, seguido de armazenamento em condição de ambiente a $22,9^{\circ} \mathrm{C}(62,3 \% \mathrm{UR})$, por até 7 dias.

\begin{tabular}{|c|c|c|c|c|c|c|c|c|c|}
\hline \multirow{3}{*}{ Período no ambiente (dia) } & \multicolumn{9}{|c|}{ Temperatura de armazenamento } \\
\hline & \multicolumn{3}{|c|}{$2^{\circ} \mathrm{C}$} & \multicolumn{3}{|c|}{$\mathbf{5}^{\circ} \mathrm{C}$} & \multicolumn{3}{|c|}{$12^{\circ} \mathrm{C}$} \\
\hline & 7 & 14 & 21 & 7 & 14 & 21 & 7 & 14 & 21 \\
\hline 1 & 7 & 8 & 8 & 6 & 8 & 8 & 1 & 1 & 1 \\
\hline 3 & 7 & 8 & 8 & 6 & 8 & 8 & 1 & 1 & 1 \\
\hline 5 & 7 & 8 & 8 & 6 & 8 & 8 & 1 & 1 & 1 \\
\hline 7 & 7 & 8 & 8 & 7 & 8 & 8 & 1 & 1 & 1 \\
\hline
\end{tabular}

TABELA 2 - Podridão, coloração da casca e da polpa e firmeza de mangas 'Palmer' armazenadas a $2^{\circ} \mathrm{C}$ ( $75,7 \%$ UR), $5^{\circ} \mathrm{C}\left(73,8 \%\right.$ UR) e $12^{\circ} \mathrm{C}(82 \%$ UR) por 7,14 e 21 dias, seguido de armazenamento em condição de ambiente a $22,9^{\circ} \mathrm{C}(62,3 \% \mathrm{UR})$.

\begin{tabular}{|c|c|c|c|c|c|c|c|c|}
\hline Temperatura (Temp) & Podridão ${ }^{1}$ & L_c $\mathbf{c}^{1}$ & Hue_c ${ }^{1}$ & Croma_c ${ }^{1}$ & $\mathbf{L} \_\mathbf{p}^{1}$ & Hue_p ${ }^{1}$ & Croma_p ${ }^{1}$ & Firmeza $^{1}$ \\
\hline $2{ }^{\circ} \mathrm{C}$ & $18,06 \mathrm{~b}$ & $40,64 \mathrm{c}$ & $59,26 \mathrm{~b}$ & $14,69 \mathrm{c}$ & $80,13 \mathrm{a}$ & $98,81 \mathrm{a}$ & $39,19 \mathrm{~b}$ & $94,80 \mathrm{a}$ \\
\hline $5^{\circ} \mathrm{C}$ & $19,44 \mathrm{~b}$ & $42,48 \mathrm{~b}$ & $64,38 \mathrm{ab}$ & $17,97 \mathrm{~b}$ & $79,02 \mathrm{~b}$ & $97,31 \mathrm{~b}$ & $41,70 \mathrm{a}$ & $82,90 \mathrm{~b}$ \\
\hline $12^{\circ} \mathrm{C}$ & $51,39 a$ & $46,57 \mathrm{a}$ & $67,22 \mathrm{a}$ & $23,56 \mathrm{a}$ & $75,78 \mathrm{c}$ & $93,74 \mathrm{c}$ & $42,74 \mathrm{a}$ & $52,42 \mathrm{c}$ \\
\hline $\mathbf{F}$ & $* *$ & $* *$ & $*$ & $* *$ & $* *$ & $* *$ & $* *$ & $* *$ \\
\hline \multicolumn{9}{|l|}{ Tempo sob refrigeraçâo } \\
\hline $\begin{array}{l}\text { (Trefri) } \\
7 \text { dias }\end{array}$ & $15,28 \mathrm{~b}$ & $40,42 \mathrm{~b}$ & $43,56 \mathrm{~b}$ & $17,26 \mathrm{~b}$ & $78,48 \mathrm{a}$ & $98,05 \mathrm{a}$ & $40,20 \mathrm{a}$ & 83,99 a \\
\hline 14 dias & $20,83 \mathrm{~b}$ & $44,57 \mathrm{a}$ & $75,67 \mathrm{a}$ & $19,96 \mathrm{a}$ & $78,60 \mathrm{a}$ & $95,79 \mathrm{~b}$ & $41,68 \mathrm{a}$ & $78,84 \mathrm{a}$ \\
\hline 21 dias & $52,78 \mathrm{a}$ & $44,70 \mathrm{a}$ & $71,63 \mathrm{a}$ & $18,99 \mathrm{ab}$ & $77,84 \mathrm{a}$ & $96,02 \mathrm{~b}$ & $41,74 \mathrm{a}$ & $67,30 \mathrm{~b}$ \\
\hline $\mathbf{F}$ & $* *$ & $* *$ & $* *$ & $*$ & NS & $* *$ & NS & $* *$ \\
\hline \multirow{2}{*}{\multicolumn{9}{|c|}{$\begin{array}{c}\text { Tempo ao ambiente } \\
\text { (Tamb) }\end{array}$}} \\
\hline & & & & & & & & \\
\hline 1 dia & $3,70 \mathrm{~b}$ & 43,19 a & 66,79 a & $16,54 \mathrm{~b}$ & $82,59 \mathrm{a}$ & $100,79 \mathrm{a}$ & $35,52 \mathrm{c}$ & $116,39 a$ \\
\hline 3 dias & $16,67 \mathrm{~b}$ & $42,87 \mathrm{a}$ & $62,72 \mathrm{a}$ & $15,80 \mathrm{~b}$ & $80,01 \mathrm{~b}$ & $99,93 \mathrm{a}$ & $38,17 \mathrm{c}$ & $91,36 \mathrm{~b}$ \\
\hline 5 dias & $40,74 \mathrm{a}$ & $43,76 \mathrm{a}$ & $64,23 \mathrm{a}$ & $20,47 \mathrm{a}$ & $77,94 \mathrm{c}$ & $96,58 \mathrm{~b}$ & $42,15 \mathrm{~b}$ & $72,97 \mathrm{c}$ \\
\hline 7 dias & $57,41 \mathrm{a}$ & $43,10 \mathrm{a}$ & $60,74 \mathrm{a}$ & $22,15 \mathrm{a}$ & $72,69 d$ & $89,18 \mathrm{c}$ & $49,00 \mathrm{a}$ & $26,11 \mathrm{~d}$ \\
\hline $\mathbf{F}$ & $* *$ & NS & NS & $* *$ & $* *$ & $* *$ & $* *$ & $* *$ \\
\hline \multicolumn{9}{|l|}{ Interações } \\
\hline Temp x Trefri & NS & $* *$ & NS & $* *$ & $* *$ & $* *$ & $* *$ & $* *$ \\
\hline Temp x Tamb & NS & $* *$ & NS & NS & $* *$ & $* *$ & NS & $* *$ \\
\hline Trefri x Tamb & $* *$ & NS & $* *$ & NS & NS & $* *$ & $* *$ & $* *$ \\
\hline Temp x Trefri x Tamb & NS & NS & NS & NS & $* *$ & $*$ & NS & $* *$ \\
\hline
\end{tabular}

${ }^{1}$ Podridão (\%); L_c = luminosidade da casca; Hue_c = ângulo hue da casca; Croma_c = cromaticidade da casca; L_p = luminosidade da polpa; Hue $\mathrm{p}=$ ângulo hue da polpa; Croma $\mathrm{p}=$ cromaticidade da polpa; Firmeza $(\mathrm{N})$.

Médias seguidas de, pelo menos, uma letra comum, na coluna, não diferem significativamente entre si, pelo teste de Tukey. NS = não significativo; ${ }^{*}=$ significativo ao nível de $1 \%$ de probabilidade; $*$ = significativo ao nível de $5 \%$ de probabilidade. 

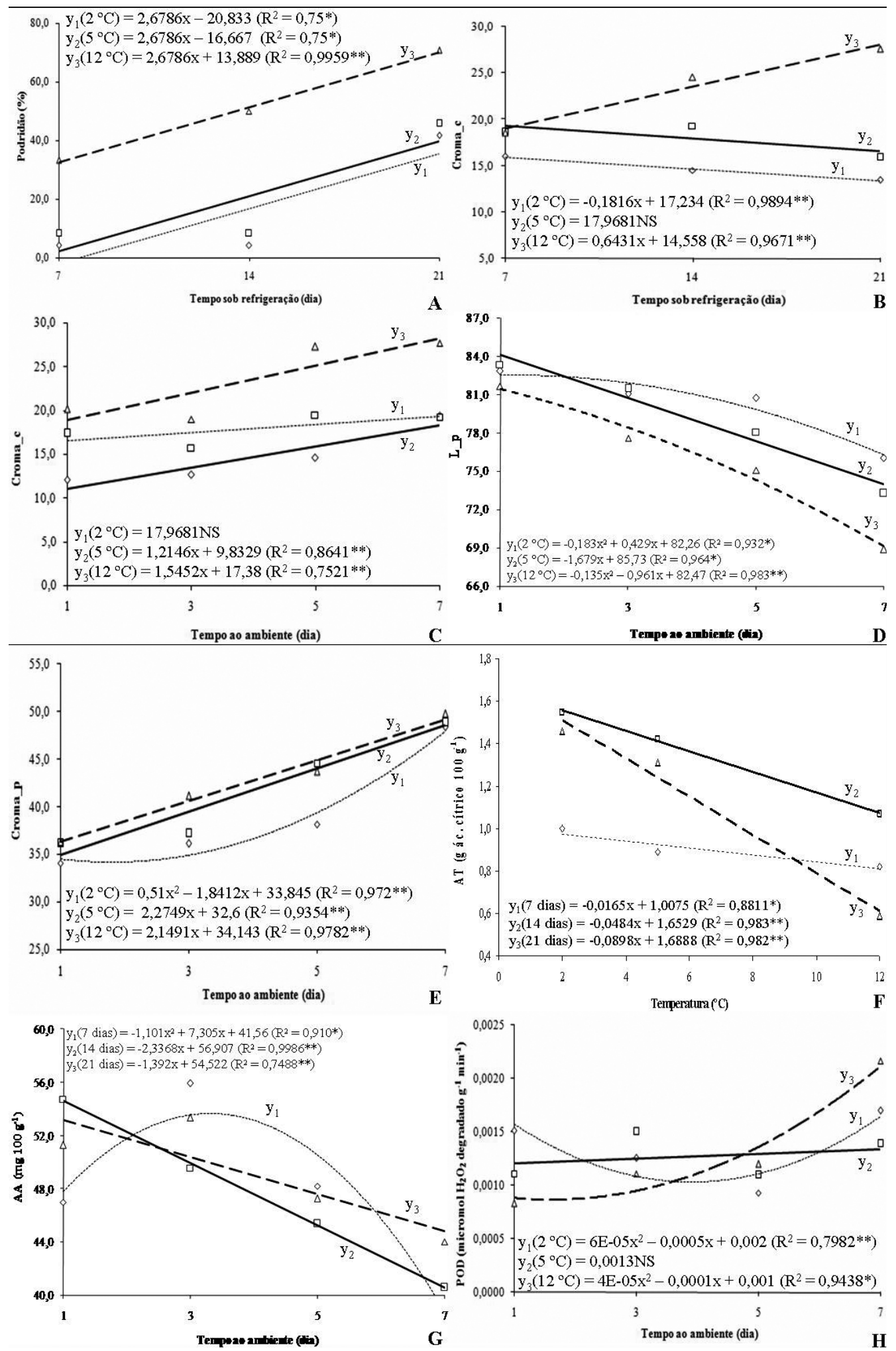

FIGURA 1 - Variação nos atributos de qualidade em mangas 'Palmer' armazenadas a $2^{\circ} \mathrm{C}(75,7 \% \mathrm{UR}), 5^{\circ} \mathrm{C}$ $\left(73,8 \%\right.$ UR) e $12^{\circ} \mathrm{C}(82 \%$ UR) por $7 ; 14$ e 21 dias, seguido de armazenamento em condição de ambiente a $22,9^{\circ} \mathrm{C}(62,3 \% \mathrm{UR})$. 
TABELA 3 - Desdobramento das interações dos parâmetros ângulo Hue (Hue_p) e firmeza da polpa em mangas 'Palmer' armazenadas a $2^{\circ} \mathrm{C}(75,7 \%$ UR $), 5^{\circ} \mathrm{C}(73,8 \%$ UR $)$ e $12^{\circ} \mathrm{C}(82 \%$ UR) por $7 ; 14$ e 21 dias, seguido de armazenamento em condição de ambiente a $22,9^{\circ} \mathrm{C}(62,3 \%$ UR).

\begin{tabular}{|c|c|c|c|}
\hline \multicolumn{4}{|c|}{${ }^{\circ}$ Hue da polpa } \\
\hline \multirow{2}{*}{ Temperatura de armazenamento } & \multicolumn{3}{|c|}{ Tempo sob refrigeração (dias) } \\
\hline & 7 & 14 & 21 \\
\hline $2^{\circ} \mathrm{C}$ & $99,11 \mathrm{aA}$ & $98,22 \mathrm{aA}$ & 99,09 aA \\
\hline $5^{\circ} \mathrm{C}$ & $97,44 \mathrm{aA}$ & $96,53 \mathrm{aA}$ & $97,96 \mathrm{aA}$ \\
\hline $12^{\circ} \mathrm{C}$ & $97,59 \mathrm{aA}$ & $92,62 \mathrm{bB}$ & $91,01 \mathrm{bB}$ \\
\hline \multirow{2}{*}{ Tempo ao ambiente (dias) } & \multicolumn{3}{|c|}{ Temperatura de armazenamento } \\
\hline & $2^{\circ} \mathrm{C}$ & $5^{\circ} \mathrm{C}$ & $12^{\circ} \mathrm{C}$ \\
\hline 1 & $101,09 \mathrm{aA}$ & $101,86 \mathrm{aA}$ & $99,42 \mathrm{aA}$ \\
\hline 3 & $101,97 \mathrm{aA}$ & $100,86 \mathrm{aA}$ & $96,97 \mathrm{bAB}$ \\
\hline 5 & $99,28 \mathrm{aA}$ & $96,12 \mathrm{bB}$ & $94,33 \mathrm{bB}$ \\
\hline 7 & $92,89 \mathrm{aB}$ & $90,41 \mathrm{aC}$ & $84,24 \mathrm{bC}$ \\
\hline \multirow{2}{*}{ Tempo ao ambiente (dias) } & \multicolumn{3}{|c|}{ Tempo sob refrigeração (dias) } \\
\hline & 7 & 14 & 21 \\
\hline 1 & $103,12 \mathrm{aA}$ & $100,22 \mathrm{bA}$ & $99,03 \mathrm{bA}$ \\
\hline 3 & $103,19 \mathrm{aA}$ & $96,95 \mathrm{cB}$ & $99,65 \mathrm{bAB}$ \\
\hline 5 & $96,86 \mathrm{aB}$ & $96,66 \mathrm{aB}$ & $96,21 \mathrm{aB}$ \\
\hline 7 & $89,01 \mathrm{aC}$ & $89,34 \mathrm{aC}$ & $89,19 \mathrm{aC}$ \\
\hline \multicolumn{4}{|c|}{ Firmeza (N) } \\
\hline \multirow{2}{*}{ Temperatura de armazenamento } & \multicolumn{3}{|c|}{ Tempo sob refrigeração (dias) } \\
\hline & 7 & 14 & 21 \\
\hline $2^{\circ} \mathrm{C}$ & $75,79 \mathrm{bA}$ & $103,33 \mathrm{aA}$ & $105,28 \mathrm{aA}$ \\
\hline $5^{\circ} \mathrm{C}$ & $66,46 \mathrm{cAB}$ & $81,83 \mathrm{bB}$ & $100,41 \mathrm{aA}$ \\
\hline $12^{\circ} \mathrm{C}$ & $59,64 \mathrm{aB}$ & $51,34 \mathrm{abC}$ & $46,29 \mathrm{bB}$ \\
\hline \multirow{2}{*}{ Tempo ao ambiente (dias) } & \multicolumn{3}{|c|}{ Temperatura de armazenamento } \\
\hline & $2^{\circ} \mathrm{C}$ & $5^{\circ} \mathrm{C}$ & $12^{\circ} \mathrm{C}$ \\
\hline 1 & $126,88 \mathrm{aA}$ & $124,97 \mathrm{aA}$ & $97,34 \mathrm{bA}$ \\
\hline 3 & $109,99 \mathrm{aB}$ & $104,75 \mathrm{aB}$ & $59,32 \mathrm{bB}$ \\
\hline 5 & $105,62 \mathrm{aB}$ & $72,59 \mathrm{bC}$ & $40,69 \mathrm{cC}$ \\
\hline 7 & $36,70 \mathrm{aC}$ & $29,29 \mathrm{aD}$ & $12,34 \mathrm{bD}$ \\
\hline \multirow{2}{*}{ Tempo ao ambiente (dias) } & \multicolumn{3}{|c|}{ Tempo sob refrigeração (dias) } \\
\hline & 7 & 14 & 21 \\
\hline 1 & $119,57 \mathrm{aA}$ & $126,44 \mathrm{aA}$ & $103,17 \mathrm{AB}$ \\
\hline 3 & $77,62 \mathrm{bB}$ & $88,02 \mathrm{bB}$ & $108,43 \mathrm{~A}$ \\
\hline 5 & $55,81 \mathrm{cC}$ & $72,27 \mathrm{bC}$ & $90,82 \mathrm{aB}$ \\
\hline 7 & $16,19 \mathrm{bD}$ & $28,61 \mathrm{abD}$ & $33,54 \mathrm{aC}$ \\
\hline
\end{tabular}

Médias seguidas da mesma letra, maiúscula nas colunas e minúscula nas linhas, não diferem entre si, pelo teste de Tukey, ao nível de $1 \%$ de probabilidade. 
TABELA 4 - Teores de sólidos solúveis (SS), acidez titulável (AT) e ácido ascórbico (AA), assim como atividade das enzimas peroxidase (POD), fenilalanina amônia-liase (PAL) e polifenoloxidase (PPO) na polpa de mangas 'Palmer' armazenadas a $2{ }^{\circ} \mathrm{C}(75,7 \%$ UR $), 5{ }^{\circ} \mathrm{C}(73,8 \%$ UR) e $12{ }^{\circ} \mathrm{C}(82 \%$ UR) por $7 ; 14$ e 21 dias, seguido de armazenamento em condição de ambiente a $22,9^{\circ} \mathrm{C}(62,3 \% \mathrm{UR})$.

\begin{tabular}{|c|c|c|c|c|c|c|}
\hline Temperatura (Temp) & $\mathbf{S S}^{1}$ & $\mathbf{A T}^{\mathbf{1}}$ & $\mathbf{A A}^{\mathbf{1}}$ & POD $^{1}$ & PAL $^{1}$ & PPO $^{1}$ \\
\hline $2{ }^{\circ} \mathrm{C}$ & $9,93 \mathrm{~b}$ & $1,336 \mathrm{a}$ & $47,49 \mathrm{a}$ & $0,0014 \mathrm{a}$ & $64,87 \mathrm{~b}$ & $10,450 \mathrm{a}$ \\
\hline $5^{\circ} \mathrm{C}$ & $10,37 \mathrm{~b}$ & $1,213 \mathrm{~b}$ & $47,97 \mathrm{a}$ & $0,0013 \mathrm{a}$ & 86,26 a & $9,315 \mathrm{~b}$ \\
\hline $12^{\circ} \mathrm{C}$ & $12,26 \mathrm{a}$ & $0,834 \mathrm{c}$ & $48,71 \mathrm{a}$ & $0,0013 \mathrm{a}$ & $58,07 \mathrm{~b}$ & $5,750 \mathrm{c}$ \\
\hline $\mathbf{F}$ & $* *$ & $* *$ & NS & NS & $* *$ & $* *$ \\
\hline \multicolumn{7}{|c|}{ Tempo de refrigeração (Trefri) } \\
\hline 7 dias & $10,95 \mathrm{a}$ & $0,906 \mathrm{c}$ & $47,65 \mathrm{a}$ & $0,0010 \mathrm{~b}$ & $64,85 \mathrm{~b}$ & $4,985 \mathrm{c}$ \\
\hline 14 dias & $10,95 \mathrm{a}$ & 1,349 a & $47,56 \mathrm{a}$ & $0,0012 \mathrm{~b}$ & 90,29 a & $8,683 \mathrm{~b}$ \\
\hline 21 dias & $10,67 \mathrm{a}$ & $1,127 \mathrm{~b}$ & $48,95 \mathrm{a}$ & $0,0017 \mathrm{a}$ & $54,05 \mathrm{c}$ & $11,848 \mathrm{a}$ \\
\hline $\mathbf{F}$ & NS & $* *$ & NS & $* *$ & $* *$ & $* *$ \\
\hline \multicolumn{7}{|l|}{ Tempo ao ambiente (Tamb) } \\
\hline 1 dia & $7,51 \mathrm{~d}$ & $1,453 \mathrm{a}$ & $51,00 \mathrm{a}$ & $0,0011 \mathrm{~b}$ & $86,18 \mathrm{a}$ & $3,472 \mathrm{~d}$ \\
\hline 3 dias & $9,91 \mathrm{c}$ & $1,333 \mathrm{~b}$ & $52,93 \mathrm{a}$ & $0,0013 \mathrm{~b}$ & $77,29 \mathrm{ab}$ & $6,234 \mathrm{c}$ \\
\hline 5 dias & $11,91 \mathrm{~b}$ & $1,071 \mathrm{c}$ & $46,93 \mathrm{~b}$ & $0,0011 \mathrm{~b}$ & $74,29 \mathrm{~b}$ & $9,834 \mathrm{~b}$ \\
\hline 7 dias & $14,092 \mathrm{a}$ & $0,653 \mathrm{~d}$ & $41,36 \mathrm{c}$ & $0,0018 \mathrm{a}$ & $41,17 \mathrm{c}$ & $14,481 \mathrm{a}$ \\
\hline $\mathbf{F}$ & $* *$ & $* *$ & $* *$ & $* *$ & $* *$ & $* *$ \\
\hline \multicolumn{7}{|l|}{ Interações } \\
\hline Temp x Trefri & $* *$ & $* *$ & NS & $*$ & $* *$ & $* *$ \\
\hline Temp x Tamb & $* *$ & NS & NS & $* *$ & $*$ & $* *$ \\
\hline Trefri x Tamb & $* *$ & $* *$ & $* *$ & NS & $* *$ & $* *$ \\
\hline Temp x Trefri x Tamb & $* *$ & $* *$ & NS & NS & $* *$ & $* *$ \\
\hline
\end{tabular}

${ }^{1} \mathrm{SS}\left({ }^{\circ} \mathrm{Brix}\right)$; AT (g ác. cítrico $\left.100 \mathrm{~g}^{-1}\right) ; \mathrm{AA}=\left(\mathrm{mg} 100 \mathrm{~g}^{-1}\right)$; POD $\left(\mu\right.$ mol de $\mathrm{H}_{2} \mathrm{O}_{2}$ degradado $\left.\mathrm{g}^{-1} \mathrm{~min}^{-1}\right)$; PAL $\left(\mathrm{nmol} \mathrm{h}^{-1} \mathrm{mg}\right.$ proteína $\left.{ }^{-1}\right)$; PPO ( $\mu$ mol de fenol degradado $\mathrm{g}^{-1} \mathrm{~min}^{-1}$ ).

Médias seguidas de pelo menos uma letra comum, na coluna, não diferem significativamente entre si, pelo teste de Tukey. NS = não significativo; ** = significativo ao nível de $1 \%$ de probabilidade; * = significativo ao nível de $5 \%$ de probabilidade.

TABELA 5 - Desdobramento das interações dos teores de sólidos solúveis $\left({ }^{\circ}\right.$ Brix $)$ da polpa de mangas 'Palmer' armazenadas a $2^{\circ} \mathrm{C}\left(75,7 \%\right.$ UR), $5^{\circ} \mathrm{C}\left(73,8 \%\right.$ UR) e $12^{\circ} \mathrm{C}(82 \%$ UR) por $7 ; 14$ e 21 dias, seguido de armazenamento em condição de ambiente a $22,9^{\circ} \mathrm{C}(62,3 \% \mathrm{UR})$.

\begin{tabular}{|c|c|c|c|}
\hline \multirow{2}{*}{ Temperatura de armazenamento } & \multicolumn{3}{|c|}{ Tempo sob refrigeração (dias) } \\
\hline & 7 & 14 & 21 \\
\hline $2^{\circ} \mathrm{C}$ & $10,50 \mathrm{aA}$ & $10,11 \mathrm{aB}$ & $9,18 \mathrm{bB}$ \\
\hline $5^{\circ} \mathrm{C}$ & $11,22 \mathrm{aA}$ & $10,38 \mathrm{abB}$ & $9,52 \mathrm{bB}$ \\
\hline $12^{\circ} \mathrm{C}$ & $11,13 \mathrm{cA}$ & $12,35 \mathrm{bA}$ & $13,32 \mathrm{aA}$ \\
\hline \multirow{2}{*}{ Tempo ao ambiente (dias) } & \multicolumn{3}{|c|}{ Temperatura de armazenamento } \\
\hline & $2^{\circ} \mathrm{C}$ & $5^{\circ} \mathrm{C}$ & $12^{\circ} \mathrm{C}$ \\
\hline 1 & $6,13 \mathrm{bD}$ & $6,46 \mathrm{bD}$ & $9,96 \mathrm{aC}$ \\
\hline 3 & $8,41 \mathrm{bC}$ & $9,04 \mathrm{bC}$ & $12,27 \mathrm{aB}$ \\
\hline 5 & $11,10 \mathrm{bB}$ & $12,07 \mathrm{abB}$ & $12,56 \mathrm{aB}$ \\
\hline 7 & $14,08 \mathrm{aA}$ & $13,92 \mathrm{aA}$ & $14,28 \mathrm{aA}$ \\
\hline \multirow{2}{*}{ Tempo ao ambiente (dias) } & \multicolumn{3}{|c|}{ Tempo sob refrigeração (dias) } \\
\hline & 7 & 14 & 21 \\
\hline 1 & $7,04 \mathrm{bD}$ & $6,99 \mathrm{bC}$ & $8,51 \mathrm{aB}$ \\
\hline 3 & $9,78 \mathrm{abC}$ & $10,71 \mathrm{aB}$ & $9,23 \mathrm{bB}$ \\
\hline 5 & $12,24 \mathrm{aB}$ & $11,52 \mathrm{aB}$ & $11,96 \mathrm{aA}$ \\
\hline 7 & $14,72 \mathrm{aA}$ & $14,57 \mathrm{aA}$ & $12,99 \mathrm{bA}$ \\
\hline
\end{tabular}

Médias seguidas da mesma letra, maiúscula nas colunas e minúscula nas linhas, não diferem entre si, pelo teste de Tukey, ao nível de $1 \%$ de probabilidade. 
TABELA 6 - Desdobramento das interações da atividade da fenilalanina amônia-liase (PAL) e da polifenoloxidase (PPO) na polpa de mangas 'Palmer' armazenadas a $2^{\circ} \mathrm{C}(75,7 \% \mathrm{UR}), 5^{\circ} \mathrm{C}$ ( $73,8 \%$ UR) e $12^{\circ} \mathrm{C}(82 \%$ UR) por $7 ; 14$ e 21 dias, seguido de armazenamento em condição de ambiente a $22,9^{\circ} \mathrm{C}(62,3 \% \mathrm{UR})$.

\begin{tabular}{|c|c|c|c|}
\hline \multicolumn{4}{|c|}{ PAL (nmol h-1 mg proteína $\left.{ }^{-1}\right)$} \\
\hline \multirow{2}{*}{ Temperatura de armazenamento } & \multicolumn{3}{|c|}{ Tempo sob refrigeração (dias) } \\
\hline & 7 & 14 & 21 \\
\hline $2^{\circ} \mathrm{C}$ & $57,81 \mathrm{bB}$ & $73,33 \mathrm{aB}$ & $63,46 \mathrm{abA}$ \\
\hline $5^{\circ} \mathrm{C}$ & $73,76 \mathrm{bA}$ & $123,42 \mathrm{aA}$ & $61,60 \mathrm{cA}$ \\
\hline $12^{\circ} \mathrm{C}$ & $62,98 \mathrm{aAB}$ & $74,11 \mathrm{aB}$ & $37,11 \mathrm{bB}$ \\
\hline \multirow{2}{*}{ Tempo ao ambiente (dias) } & \multicolumn{3}{|c|}{ Temperatura de armazenamento } \\
\hline & $2^{\circ} \mathrm{C}$ & $5^{\circ} \mathrm{C}$ & $12^{\circ} \mathrm{C}$ \\
\hline 1 & $64,39 \mathrm{bBC}$ & $100,46 \mathrm{aA}$ & $93,67 \mathrm{aA}$ \\
\hline 3 & $65,84 \mathrm{bAB}$ & $100,44 \mathrm{aA}$ & $65,60 \mathrm{bB}$ \\
\hline 5 & $79,97 \mathrm{bA}$ & $98,08 \mathrm{aA}$ & $44,81 \mathrm{cC}$ \\
\hline 7 & $49,28 \mathrm{aC}$ & $46,06 \mathrm{aB}$ & $28,18 \mathrm{bD}$ \\
\hline \multirow{2}{*}{ Tempo ao ambiente (dias) } & \multicolumn{3}{|c|}{ Tempo sob refrigeração (dias) } \\
\hline & 7 & 14 & 21 \\
\hline 1 & $70,01 \mathrm{bA}$ & $109,53 \mathrm{aA}$ & $78,99 \mathrm{bA}$ \\
\hline 3 & $61,30 \mathrm{bA}$ & $104,74 \mathrm{aA}$ & $65,84 \mathrm{bAB}$ \\
\hline 5 & $70,60 \mathrm{bA}$ & $100,34 \mathrm{aA}$ & $51,92 \mathrm{cB}$ \\
\hline 7 & $57,50 \mathrm{aA}$ & $46,54 \mathrm{aB}$ & $19,47 \mathrm{bC}$ \\
\hline \multicolumn{4}{|c|}{ PPO (micromol fenol degradado $\mathrm{g}^{-1} \mathrm{~min}^{-1}$ ) } \\
\hline \multirow{2}{*}{ Temperatura de armazenamento } & \multicolumn{3}{|c|}{ Tempo sob refrigeração (dias) } \\
\hline & 7 & 14 & 21 \\
\hline $2^{\circ} \mathrm{C}$ & $6,546 \mathrm{cA}$ & $9,577 \mathrm{bA}$ & $15,228 \mathrm{aA}$ \\
\hline $5^{\circ} \mathrm{C}$ & $5,106 \mathrm{cA}$ & $9,754 \mathrm{bA}$ & $13,086 \mathrm{aB}$ \\
\hline $12^{\circ} \mathrm{C}$ & $3,301 \mathrm{bB}$ & $6,718 \mathrm{aB}$ & $7,231 \mathrm{aC}$ \\
\hline \multirow{2}{*}{ Tempo ao ambiente (dias) } & \multicolumn{3}{|c|}{ Temperatura de armazenamento } \\
\hline & $2^{\circ} \mathrm{C}$ & $5^{\circ} \mathrm{C}$ & $12^{\circ} \mathrm{C}$ \\
\hline 1 & $4,422 \mathrm{aD}$ & $3,411 \mathrm{aC}$ & $2,582 \mathrm{aC}$ \\
\hline 3 & $6,988 \mathrm{aC}$ & $8,655 \mathrm{aB}$ & $3,058 \mathrm{bC}$ \\
\hline 5 & $12,125 \mathrm{aB}$ & $10,919 \mathrm{aB}$ & $6,457 \mathrm{bB}$ \\
\hline 7 & $18,267 \mathrm{aA}$ & $14,276 \mathrm{bA}$ & $10,902 \mathrm{cA}$ \\
\hline \multirow{2}{*}{ Tempo ao ambiente (dias) } & \multicolumn{3}{|c|}{ Tempo sob refrigeração (dias) } \\
\hline & 7 & 14 & 21 \\
\hline 1 & $2,378 \mathrm{bB}$ & $3,200 \mathrm{abD}$ & $4,837 \mathrm{aD}$ \\
\hline 3 & $3,389 \mathrm{cB}$ & $6,506 \mathrm{bC}$ & $8,805 \mathrm{aC}$ \\
\hline 5 & $6,095 \mathrm{cA}$ & $9,768 \mathrm{bB}$ & $13,639 \mathrm{aB}$ \\
\hline 7 & $8,075 \mathrm{cA}$ & $15,258 \mathrm{bA}$ & $20,111 \mathrm{aA}$ \\
\hline
\end{tabular}

Médias seguidas da mesma letra, maiúscula nas colunas e minúscula nas linhas, não diferem entre si, pelo teste de Tukey, ao nível de probabilidade indicado nas interações da Tabela 4. 


\section{CONCLUSÕES}

Mangas da cultivar Palmer podem ser conservadas sob refrigeração a $12^{\circ} \mathrm{C}$ por 21 dias, sem prejuízos ao amadurecimento, porém com limitações devido à ocorrência de podridões. $\mathrm{O}$ armazenamento a $2^{\circ} \mathrm{C}$ e a $5^{\circ} \mathrm{C}$ foi limitado pela ocorrência de injúrias na casca, que foram mais severos a $2^{\circ} \mathrm{C}$, com comprometimento do desenvolvimento da coloração característica da cultivar. O amadurecimento da polpa destes frutos não foi prejudicado pela ocorrência de injúrias na casca, mas ocorreu com menor intensidade que nos frutos armazenados a $12^{\circ} \mathrm{C}$.

\section{AGRADECIMENTOS}

À Fundação de Amparo à Pesquisa do Estado de São Paulo (FAPESP), pelo auxílio financeiro (Proc. $n^{\circ}$ 09/51977-9).

\section{REFERÊNCIAS}

ALVES, R. E.; FILGUEIRAS, H. A. C.; MENEZES, J. B.; ASSIS, J. S. de; LIMA, M. A. C. de; AMORIM, T. B. F.; MARTINS, A. G. Colheita e Pós-colheita. In: GENÚ, P. J. C.; PINTO, A. C. Q. (Ed.). A cultura da mangueira. Brasília: Embrapa Informação Tecnológica, 2002. p.383-405.

AOAC. Official methods of analysis. $18^{\text {th }}$ ed. Gaithersburg, 2005. chap. 37; 42, p.10-11.

BERNARDES-SILVA, A. P. F.; LAJOLO, F. M.; CORDENUSSI, B. R. Evolução dos teores de amido e açúcares solúveis durante o desenvolvimento e amadurecimento de diferentes cultivares de manga. Ciência e Tecnologia de Alimentos, Campinas, v. 23, p. 116-120, 2003. Suplemento.

CAHILL, D. M.; McCOMB, J. A. A comparison of changes in phenylalanine ammonia-lyase activity, lignin and phenolic synthesis in the roots of Eucalyptus calophylla (field resistant) and E. marginata (susceptible) when infected with Phytophthora cinnamomi. Physiological and Molecular Plant Pathology, London, v.40, n.2, p.315-332, 1992.
CANTWELL, M. I.; KASMIRE, R. E. Postharvest handling systems: fruit vegetables. In: KADER, A. A. (Ed.). Postharvest technology of horticultural crops. Oakland: University of California, 2002. p. 407-421.

CHITARRA, M. I. F.; CHITARRA, A. B. Pós-colheita de frutas e hortaliças: fisiologia e manuseio. 2.ed. Lavras: UFLA, 2005. 785 p.

CORRÊA, M. O. G.; PINTO, D. D.; ONO, E. O. Análise da atividade respiratória em frutos de jabuticabeira. Revista Brasileira de Biociências, Porto Alegre, v.5, supl.2, p.831-833, 2007.

KADER, A. A.; ARPAIA, M. L. Postharvest handling systems: subtropical fruit. In: KADER, A. A. (Ed.). Postharvest technology of horticultural crops. $3^{\text {rd }}$ ed. Oakland: University of California, 2002. p. 375-384.

LIMA, G. P. P.; BRASIL, O. G.; OLIVEIRA, A. M. de. Poliaminas e atividade da peroxidase em feijão (Phaseolus vulgaris L.) cultivado sob estresse salino. Scientia Agricola, Piracicaba, v.56, n.1, p.21-26, 1999.

LIMA, M. A. C. de; SILVA, A. L. da; AZEVEDO, S. S. N.; SANTOS, P. de S. Tratamentos pós-colheita com 1-metilciclopropeno em manga 'Tommy Atkins': efeito de doses e número de aplicações. Revista Brasileira de Fruticultura, Jaboticabal, v.28, n.1, p. 64-68, 2006.

MAPA. Uma década de bons frutos. Informativo CGPCP Fruticultura, Brasília, v.5, n.46, p.1-7, 2011.

MENTEN, M. M. Clima favorece a mangicultura em 2010. Hortifruit Brasil, São Paulo, p. 42-43, 2010.

MIGUEL, A. C. A.; DURIGAN, J. F.; MORGADO, C. M. A.; GOMES, R. F. O. Injúria pelo frio na qualidade pós-colheita de mangas cv. Palmer. Revista Brasileira de Fruticultura, Jaboticabal, p.255-260, 2011. Volume especial.

MORAIS, P. L. D. de; ASSIS, J. S. de. Quality and conservation of mango 'Tommy Atkins' as affected by maturity stage and storage temperature. Acta Horticulturae, Leuven, v. 645, p. 639-643, 2004. 
NAIR, S.; SINGH, Z. Chilling injury during storage affects respiration rate and fruit quality in Kensington Pride mango fruit. Acta Horticulturae, Leuven, v. 820, p. 737-743, 2009.

NEVES, L. C.; BENEDETTE, R. M.; SILVA, V. X. da; VIEITES, R. L.; ROBERTO, S. R. Dano de frio em limas-ácidas Tahiti, colhidas em diferentes épocas e submetidas a tratamentos térmicos e bioquímicos. Revista Brasileira de Fruticultura, Jaboticabal, v. 30, n. 2, p. 377-384, 2008.

NUNES, M. C. N.; EMOND, J. P.; BRECHT, J. K.; DEA, S.; PROULX, E. Quality curves for mango fruit (cv. Tommy Atkins and Palmer) stored at chilling and nonchilling temperatures. Journal of Food Quality, Chicago, v. 30, p. 104-120, 2007.

PINTO, A. C. de Q.; PINHEIRO NETO, F.; GUIMARÃES, T. G., Estratégias do melhoramento genético da manga visando a atender a dinânmica de mercado. Revista Brasileira de Fruticultura, Jaboticabal, v. 33, n.1 - edição especial, p.64-72, 2011.

SANTOS, L. O. Conservação pós-colheita de mangas produzidas na região de Jaboticabal-SP. 2008. 103 f. Dissertação (Mestrado em Produção Vegetal) - Faculdade de Ciências Agrárias e Veterinárias, Universidade Estadual Paulista, Jaboticabal, 2008.

SANTOS, L. O.; DURIGAN, J. F.; MARTINS, R. N.; MORGADO, C. M. A. Conservação e qualidade de mangas 'Palmer' submetidas a tratamento com fungicidas e hidrotérmico. Ciência e Agrotecnologia, Lavras, v.34, n.6, p. 1514-1521, 2010.
STROHECKER, R.; HENNING, H. M. Analisis de vitaminas: métodos comprobados. Madrid: Paz Montalvo, 1967. $428 \mathrm{p}$.

TEIXEIRA, G. H. de A.; DURIGAN, J. F.; ALVES, R. E.; O'HARE, T. J. Use of modified atmosphere to extend shelf life of fresh-cut carambola (Averrhoa carambola L.). Postharvest Biology and Technology, Amsterdam, v. 44, n. 1, p. 80-85, 2007.

TEIXEIRA, G. H. de A.; DURIGAN, J. F. Storage of 'Palmer' mangoes in low-oxygen atmospheres. Fruits, Paris, v. 66, p. 279-289, 2011.

VASQUEZ-SALINAS, C. ; LAKSHMINARAYANA, S. Compositional changes in mango fruit during ripening at different storage temperatures. Journal of Food Science, Washington, v. 50, p. 1646-1648, 1985.

VELA, G.; LEÓN, D. M.; GARCÍA, H. S.; CRUZ, J. Polyphenoloxidase activity during ripening and chilling stress in 'Manila' mangoes. Journal of Horticultural Science \& Biotechnology, United Kingdom, v. 78, n. 1, p. 104-107, 2003.

WANG, C. Y. Physiological and biochemical responses of plants to chilling stress. HortScience, Alexandria, v. 17, p. 173-186, 1982.

WHANGCHAI, K.; GEMMA, H.; IWAHORI, S.; UTHAIBUTRA, J. Endogenous polyamines in 'Nam Dok Mai' mangoes with different ripening stages and its relation to chilling injury during storage. Acta Horticulturae, Leuven, v. 509, p. 429-433, 2000. 